KOVALEVA L.V ${ }^{\circledR}{ }^{\circledR}$, VORONKOV A.S. ${ }^{\mathbf{1}}$, ZAKHAROVA E.V. ${ }^{2}$, TIMOFEEVA G.V. ${ }^{\mathbf{1}}$

${ }^{I}$ Timiryazev Institute of Plant Physiology RAS,

Russia, Moscow, Botanicheskaya, 35

${ }^{2}$ Dep. of Genetics, Biotechnology, Plant Breeding \& Seed Science, Timiryazev Agricultural Academy,

Russia, Moscow

\kovaleva_l@mail.ru

\title{
PM $\mathrm{H}^{+}$-ATPase IS A POTENTIAL TARGET OF HORMONE SIGNALING DURING POLLEN TUBE GROWTH IN PETUNIA
}

In plants, successful fertilization involves pollen tube growth through the pistil tissue towards the egg cells. A fine-tuned network of cellular processes is required to regulate pollen grains germination and the elongation of pollen tubes. These processes include a dynamic organization of the cytoskeleton, vesicle and protein trafficking, establishing intracellular tip-localized $\mathrm{Ca}^{2+}$ and $\mathrm{pH}$ gradients and local ion fluxes generating an endogenous electrical field, adjustment of turgor pressure, an active metabolism and signal transduction pathways [1-3]. However, how the activities of these components are coordinated to properly regulate pollen tube growth remains to be largely unknown.

Earlier it has been shown that petunia pollen grain germination in vitro is accompanied by changes in the levels of plant hormones, ethylene, indolyl acetic acid (IAA), abscisic acid (ABA), gibberellins (GAs) and cytokinins, and is sensitive to the treatment by exogenous ones $[4,5]$. These and most recent data [6] allowed us to put forward a hypothesis about hormonal signaling in polar growth of pollen tubes. The purpose of the present work was to check a validity of the above hypothesis focused on the interaction of plant hormones with the key player of polar pollen tube growth, namely the $\mathrm{H}^{+}$-ATPase in the plasma membrane (PM) of male gametophyte.

PM $\mathrm{H}^{+}$-ATPase energizes pollen PM for nutrient, ion and water uptake, but additionally its activity directly affects the germination and growth of pollen tubes [7]. External as well as cytosolic signals and metabolic pathways are integrated by $\mathrm{PM} \mathrm{H}^{+}$-ATPase and directly influenced growth rates engaging $\mathrm{PM} \mathrm{H}^{+}$-ATPase to an essential node in the signaling network of pollen tubes. Certal et $a l$. [8] recently showed that proton dynamics may underlie basic mechanisms responsible for polarity and spatial regulation of pollen tube growth.

\section{Materials and methods}

Plants and treatments. Plants of petunia (Petunia hybrida L.) from our collection were propagated vegetatively in tubes on agar-solidified MS medium in the climate-controlled chamber $\left(25^{\circ} \mathrm{C}, 16-\mathrm{h}\right.$ photoperiod). 45-60 day-old plants were transferred into 51-plastic vessels with soil and grown in the greenhouse under natural illumination. All experiments were done on freshly collected mature pollen.

Pollen was cultivated for $4 \mathrm{~h}$ in thermostat at $26^{\circ} \mathrm{C}$ on the medium containing $0.4 \mathrm{M}$ sucrose and $1.6 \mathrm{mM} \mathrm{H}_{3} \mathrm{BO}_{3}$. Plant hormones, IAA, ABA, $\mathrm{GA}_{3}$, and kinetin $(1 \mu \mathrm{M})$ were added to the medium simultaneously with pollen.

The pollen tubes were examined under the microscope Axio Imedger D1 equipped with Axio Cam MRm camera. The pollen tubes length was measured using AxioVision 4.8 software (Carl Zeiss, Germany). In each treatment these measurements were made on 100 pollen tubes.

Monitoring hormone-induced changes in the membrane potential on the PM of pollen tubes. As the activity of $\mathrm{PM} \mathrm{H}^{+}$-ATPase generates transmembrane proton gradient $\left(\Delta \mu \mathrm{H}^{+}\right)$involving both $\Delta \mathrm{pH}$ and the membrane potential $(\Delta \psi)$ we attempted to elucidate whether the $\Delta \psi$ is sensitive to exogenous plant hormones. For monitoring the membrane potential on the PM of growing pollen tubes they were cultivated on the medium containing $0.4 \mathrm{M}$ sucrose and $1.6 \mathrm{mM} \mathrm{H}_{3} \mathrm{BO}_{3}$. To this end, a possible sensitivity of the $\Delta \psi$ on pollen tubes PM (negative inside) to plant hormones (IAA, $\mathrm{ABA}, \mathrm{GA}_{3}$ and kinetin) was tested with the use of cationic probe safranin $O$. The hormones at concentration of $1 \mu \mathrm{M}$ were added to suspension of growing pollen tubes preequilibrated with this probe in $\mathrm{K}^{+}$-free assay medium. Hormone-induced changes in $\Delta \Psi$ of pollen tubes were monitored by following differential absorbance $\left(\mathrm{A}_{554}-\mathrm{A}_{524}\right)$

C KOVALEVA L.V., VORONKOV A.S., ZAKHAROVA E.V., TIMOFEEVA G.V. 
changes of the dye. Spectrophotometric measurements were carried out with a doublewavelength spectrophotometer Hitachi-557 (Japan) at $25^{\circ} \mathrm{C}$ in standard $1-\mathrm{cm}$ cuvettes with continuous stirring their content with gentle air flow passing through pollen suspension in order to prevent its sedimentation in the course of experiments.

Fluorescent immunolabeling of $\mathrm{PM} \mathrm{H^{+ } -}$ ATPase. Pollen tubes were immediately fixed with $4 \%$ paraformaldehyde (PFA) in $10 \mathrm{mM}$ PBS (PBS: $2.68 \mathrm{mM} \mathrm{KCl}, 0.15 \mathrm{M} \mathrm{Na}_{2} \mathrm{HPO}_{4}$, and $0.086 \mathrm{M}$ $\mathrm{KH}_{2} \mathrm{PO}_{4}$ ) overnight at $4^{\circ} \mathrm{C}$. Samples were then washed in a regular salt rinse solution (RSRS: 10 $\mathrm{mM}$ PBS, $0.15 \mathrm{M} \mathrm{NaCl}, 0.1 \%$ (vol/vol) Tween 20 and $0.8 \% \mathrm{BSA}$ ) and washed with $10 \mathrm{mM}$ PBS containing $0.8 \%$ BSA solution (PBS+BSA) to remove the Tween 20. After the application of rabbit anti- $\mathrm{H}^{+}$-ATPase antibodies (Agrisera, AS07 260; 1:300 dilutions in $10 \mathrm{mM}$ PBS, $1 \%$ BSA, $\mathrm{pH}$ 7.2), samples were incubated for $4 \mathrm{~h}$ at $37^{\circ} \mathrm{C}$. After hybridization samples were subjected to a series of vigorous washes, twice with a highsalt rinse solution (HSRS: $10 \mathrm{mM}$ PBS, $0.5 \mathrm{M}$ $\mathrm{NaCl}, 0.1 \%$ (vol/vol) Tween 20 and $0.1 \%$ BSA) for $10 \mathrm{~min}$, once with RSRS for $10 \mathrm{~min}$, and washed with PBS+BSA. The chiken anti-rabbit $\lg G$ (HL), DyLight 488 conjugated antibodies (Agrisera, AS10 831; 1:500 dilutions in $10 \mathrm{mM}$ PBS, $0.15 \mathrm{M} \mathrm{NaCl}, 1 \% \mathrm{BSA}, \mathrm{pH}$ 7.2) was incubated overnight at room temperature. After washing with RSRS twice for $5 \mathrm{~min}$, specimens were mounted, covered with a coverslip, and observed under fluorescent microscope
(AxioImager Z2 with ApoTome, AxioCam MRm camera; filter set $65 \mathrm{HE} \mathrm{-} \mathrm{excitation} \mathrm{475/30,}$ emission 550/100; Carl Zeiss, Germany). Using AxioVision 4.8, the image of pollen tube was divided on 8 equal $10 \mu \mathrm{m}$ zones initiating from the tip, thereafter the profile reflecting distribution of fluorescence intensity on all pollen tube zones was constructed. At least 50 pollen tubes were monitoring in each of five replicates. The mean fluorescence intensity of DyLight 488 in each pollen tube zone was calculated using Microsoft office Excel 2007 software.

\section{Results and discussion}

Effects of plant hormones on pollen tube growth. In the present work, in order to compare effects of all the exogenous hormones under study they were used at concentration of $1 \mu \mathrm{M}$. As follows from Fig. 1, IAA, ABA and $\mathrm{GA}_{3}$ at this concentration stimulated the process in question, while kinetin, in contrast, inhibited pollen tube growth.

Effects of plant hormones on the membrane potential on pollen tube PM. An addition of IAA to pollen tube suspension immediately initiated their membrane hyperpolarization, as judged by fluorescence quenching of safranin $\mathrm{O}$ related to inward flow of the dye across pollen tube PM, and this effect achieved a saturation level in approximately $10-15 \mathrm{~min}$ (Fig. $2 \mathrm{a}$ ). In fact, a similar effect took place also in the presence of $\mathrm{ABA}$ and $\mathrm{GA}_{3}$ (Fig. $2 \mathrm{c}, \mathrm{j}$ ).

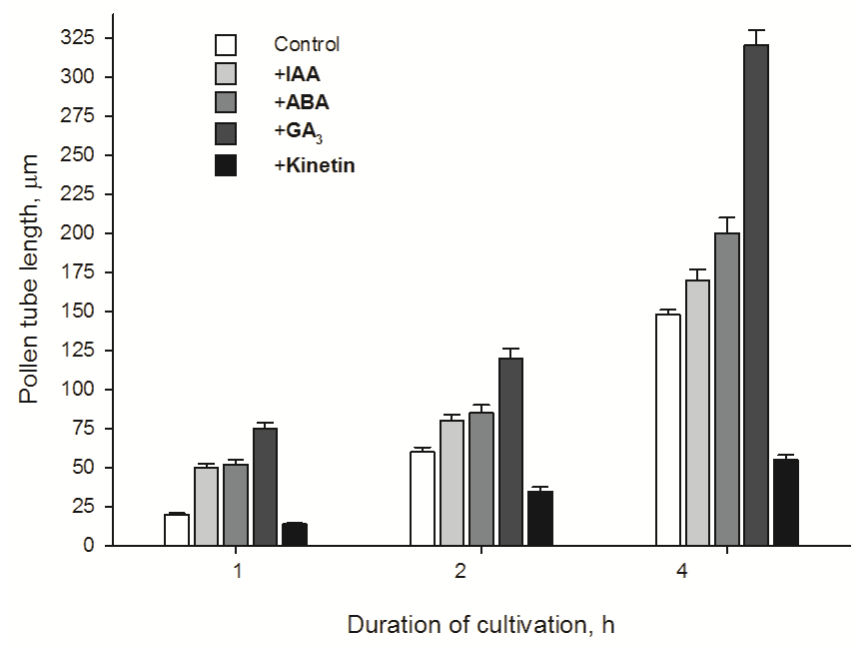

Fig. 1. Effects of plant hormones on petunia pollen tube growth on medium containing $0.4 \mathrm{M}$ sucrose $+1.6 \mathrm{mM}$ $\mathrm{H}_{3} \mathrm{BO}_{3}$. Data represent the mean from independent experiments $(\mathrm{n}=6-10)$. Result significance was assessed with the Student's $t$-Test at $P<0.05$. 
Reversal of the hormone-induced fluorescence quenching due to leakage of the dye was observed after addition of $60 \mathrm{mM} \mathrm{KCl}$ to pollen tubes suspension as a result of the depolarization of the pollen membrane (Fig. 2 a, c, j). Subsequent addition of valinomycin had practically no effect on the $\mathrm{K}^{+}$-induced dissipation of the membrane potential (unpublished data) indicating relatively high intrinsic potassium permeability of the pollen PM. Here, it is important that the fast enough response of the fluorescent signal to change in the external $\mathrm{K}^{+}$concentration shows that the observed dye response most likely reflects the changes in the PM polarization rather than those in the membrane potential on any intracellular membranes of pollen grains/tubes. This can be considered as a suitable approach to specifically distinguish the total fluorescence signal from the membrane potential induced by dye response to the PM energization. The hormone-induced hyperpolarization of pollen tubes was also observed in the similar experiments carried out with DiS- $\mathrm{C}_{3}-(5)$ (unpublished data).
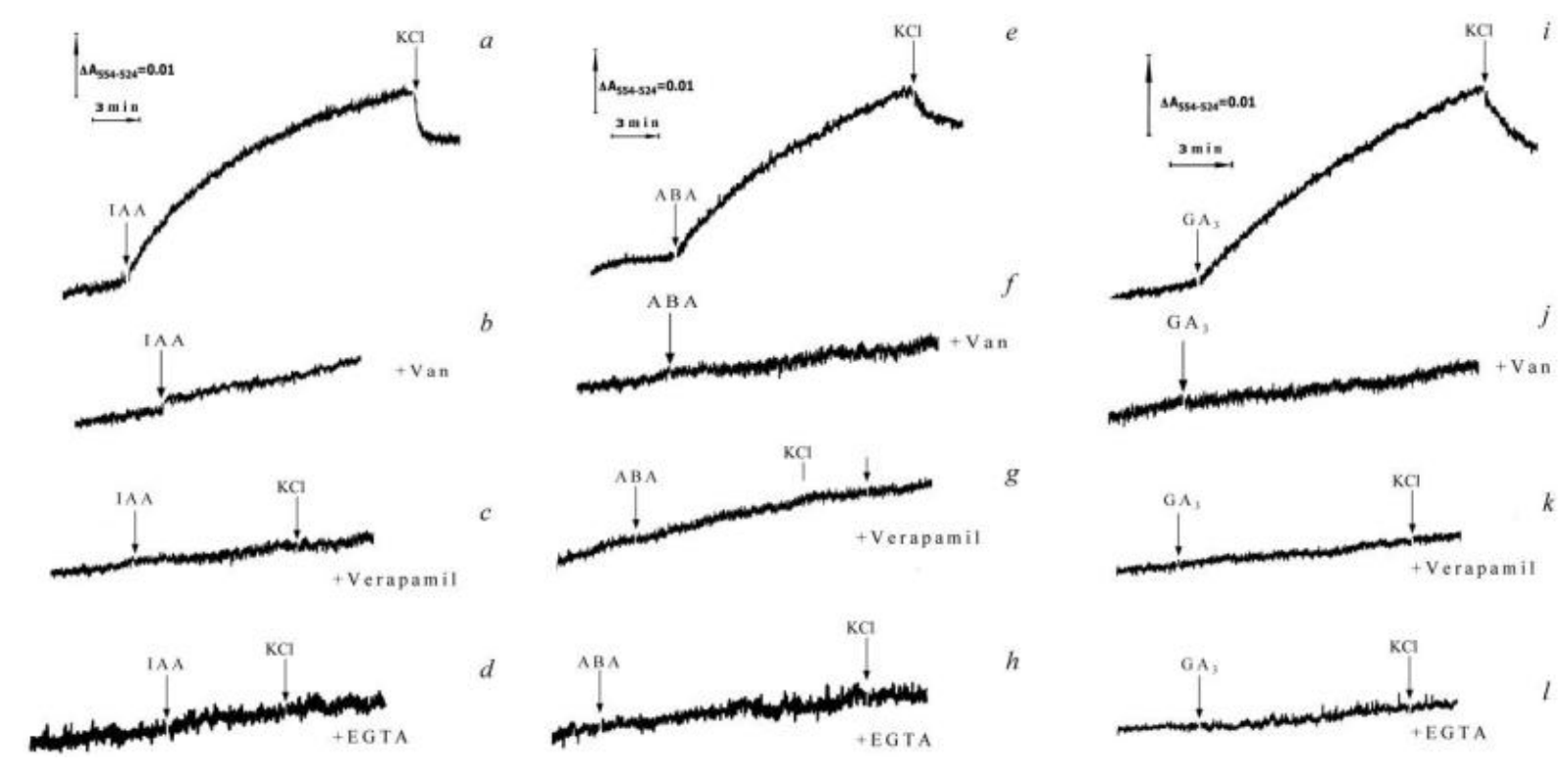

Fig. 2. Hyperpolarization of the PM of petunia pollen tubes triggered by plant hormones and assayed by the potential-sensitive dye safranin O.O. Where indicated, $1 \mu \mathrm{M}$ IAA (a), $1 \mu \mathrm{M}$ ABA (c) and $1 \mu \mathrm{M} \mathrm{GA}$ (j), $60 \mathrm{mM} \mathrm{KCl}$, $100 \mu \mathrm{M}$ verapamil $(\mathrm{c}, \mathrm{g}, \mathrm{k})$ and $1 \mathrm{mM}$ EGTA $(\mathrm{d}, 1, \mathrm{i})$ were added to pollen tube suspension in the $\mathrm{K}^{+}$-free assay medium containing $10 \mu \mathrm{M}$ safranin $\mathrm{O}$. In figures, representative kinetic curves from three independent experiments replicated at least five times are shown.

To highlight the contribution of $\mathrm{H}^{+}$-ATPase pumps to the PM hyperpolarization due to plant hormones, we used orthovanadate, a well known specific inhibitor of plant $\mathrm{PM} \mathrm{H}^{+}$-ATPase. Application of $200 \mu \mathrm{M}$ orthovanadate completely abolished the effect caused by IAA or ABA or $\mathrm{GA}_{3}$ (Fig. $2 \mathrm{~b}, \mathrm{f}, \mathrm{j}$ ). These findings indicate that the proton pump was active, had an electrogenic component and most likely is involved in the hormone-induced hyperpolarization of the PM. The significant contribution of $\mathrm{H}^{+}$-ATPase to this effect is also supported by its stimulation by protonophore FCCP as well as by $\mathrm{K}^{+} / \mathrm{H}^{+}$exchanger nigericin (unpublished data) due to the conversion of transmembrane $\Delta \mathrm{pH}$ into $\Delta_{\Psi}$ during the operation of proton pump. The observed inhibitory action of
EGTA related to dramatic decline of $\mathrm{Ca}^{2+}$ level in the external medium may be attributed to prevention of external calcium entry into the pollen tubes through the PM. This interpretation is supported, as revealed here, by a qualitatively similar inhibiting effect also induced by verapamil. As shown in Fig. $2 \mathrm{~d}, 1, \mathrm{i}$, this calcium channel blocker abolished completely both the IAA-, ABAand $\mathrm{GA}_{3}$-induced hyperpolarization of the $\mathrm{PM}$ of growing pollen tubes thereby resembling the effect caused by EGTA.

Kinetin has no effect on the membrane potential of pollen tubes.

Effects of plant hormones on lateral allocation of pollen tube PM $\mathrm{H}^{+}$-ATPase. Taking into account the above results concerning the 
hormone-induced stimulation of electrogenic

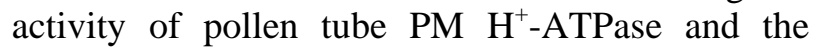
recent data that this enzyme is excluded from the tip of growing pollen tubes [8], the possibility arose that the stimulatory hormone effects are accompanied by the change in lateral membrane allocation of the proton pump as well. In order to test this possibility pollen $\mathrm{PM} \mathrm{H}^{+}$-ATPase was labeled with fluorescent anti- $\mathrm{H}^{+}$-ATPase antibodies. In the control test, pollen tubes were cultivated for $1 \mathrm{~h}$ in the standard medium and thereafter they were treated with anti- $\mathrm{H}^{+}$-ATPase

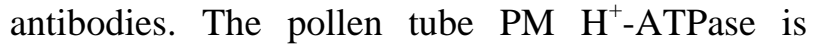
mainly localized in the zone $10-20 \mu \mathrm{m}$ from the tip of the tube as follows from the pattern of lateral fluorescent label distribution in the tube PM (Fig. 3 a).

As follows from Fig. 3 (b and c), pollen tubes cultivated in the same medium in the presence of $1 \mu \mathrm{M}$ IAA or ABA displayed a different fluorescent label distribution in pollen tube PM indicating preferred localization of PM $\mathrm{H}^{+}$-ATPase in zone $20-40 \mu \mathrm{m}$ from the tip of tubes. The effect of ABA appeared to be most evident (Fig. $3 \mathrm{c}, \mathrm{f}$ and 4). Pollen tubes treated with $1 \mu \mathrm{M}$ $\mathrm{GA}_{3}$ and kinetin, unlike those treated with IAA or ABA, did not display clear response of the fluorescent label distribution in the pollen tube PM, and only slight enough fluorescence was observed along the entire length of pollen tube (Fig. 3 d, e and 4).
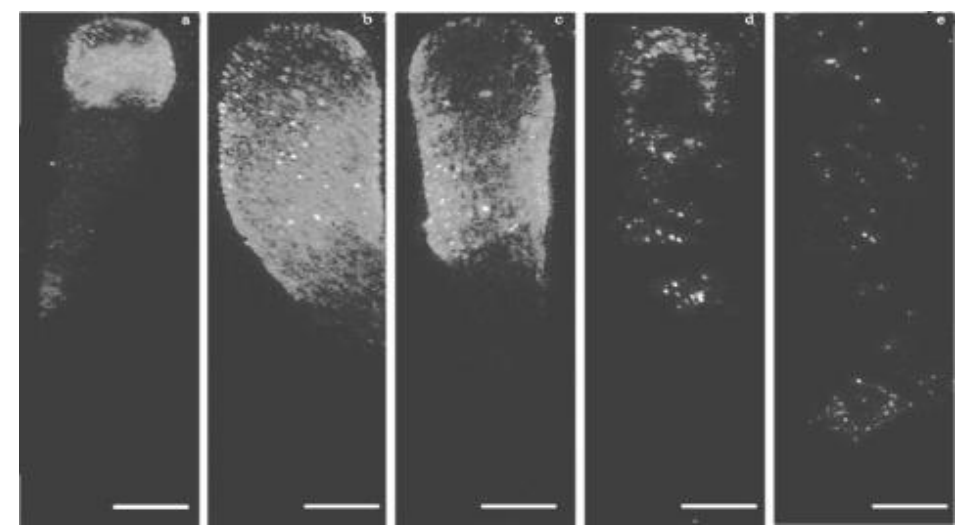

Fig. 3. Effects of IAA, ABA, $\mathrm{GA}_{3}$ and kinetin on $\mathrm{PM}$ allocation of the $\mathrm{PM} \mathrm{H}^{+}$-ATPase in petunia pollen tubes labeled with DyLight 488 and examined under AxioImager Z2 with ApoTome. The plant hormones were added to pollen tubes after their incubation in cultivation medium for $2 \mathrm{~h}$. Primary antibodies were Rabbit anti- $\mathrm{H}^{+}$-ATPase (Agrisera, AS07 260) while secondary antibodies were Chiken antirabbit IgG (HL), DyLight 488 conjugated (Agrisera, AS10 831). Scale bar $=10 \mu \mathrm{m}$.

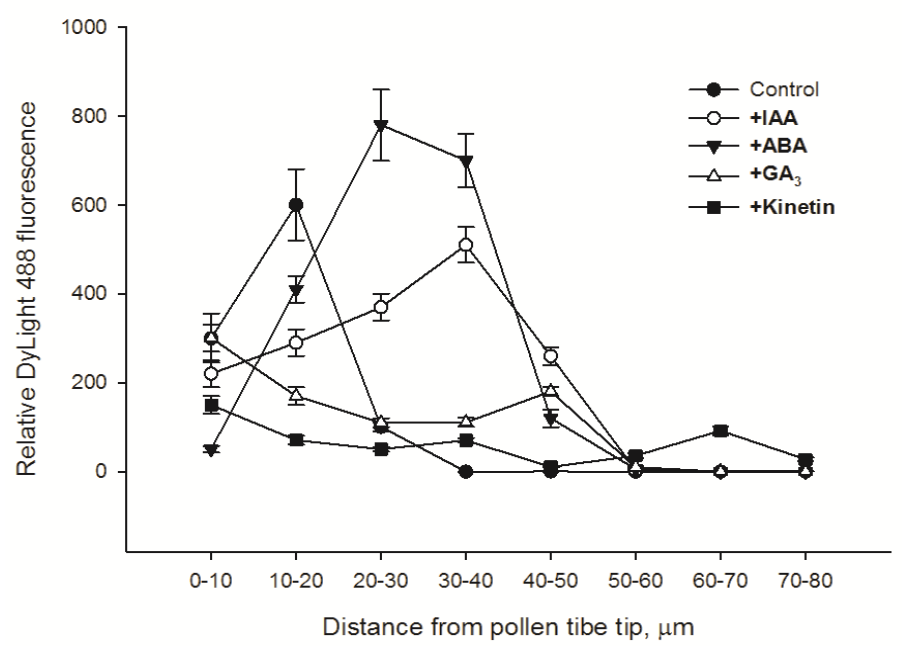

Fig. 4. Effects of IAA, ABA, $\mathrm{GA}_{3}$ and kinetin on relative DyLight 488 fluorescence intensity measured using AxioVision 4.8 software. At least 50 pollen tubes were used in each experiment of five replicates and corresponding mean fluorescence intensities were calculated. Data are means \pm SD obtained in three independent experiments with ten replicates in each one. Difference significance was assessed with the Student's $t$-test at $P<0.05$. 


\section{Conclusions}

1) The important conclusion is that only in the case of auxin all the observed hormone-induced responses of growing PTs may be integrated into a common mechanism, in which $\mathrm{Ca}^{2+}$ ions can putatively serve as coordinative and integrating signal. IAA exerted the strongest effect on F-actin [6] thereby suggesting the involvement of this hormone in the regulation of actin filaments (AF) assembly through corresponding modulation of the activity of $\mathrm{pH}$-sensitive ABPs. Therefore, it can be concluded that auxin plays a key role in the maintenance of pollen tube polar growth that is in accordance with its similar behavior in other plant organs.
2) The inhibitory effect of kinetin on pollen tube growth was related to degradation of F-actin along the length of the pollen tube [6]. Apparently, both auxin and cytokinin play an important role in the regulation of the actin cytoskeleton during pollen tube growth via their effects on actin polymerization. The hypothesis about the interaction of these hormones when applied to cytoskeleton has been formulated long ago [9].

3) Although $\mathrm{GA}_{3}$ displayed the strongest stimulating action on petunia pollen tube growth, it, unlike IAA, practically had no any effect on the lateral membrane allocation of the pollen $\mathrm{PM} \mathrm{H}^{+}$ATPase.

This work was supported by grant from the Russian Foundation for Basic Research (RFBR, no. 10-04-00356 and 13-04-00592).

\section{References}

1. Feijo J.A., Malho R., Obermeyer G. Ion dynamics and its possible role during in vitro pollen germination and tube growth // Protoplasma. - 1995. - V. 187. - P. 155-167.

2. Holdaway-Clarke T.L., Hepler P.K. Control of pollen tube growth: role of ion gradients and fluxe // New Phytol. - 2003. V. 159. - P. 539-563.

3. Hepler P.K. The cytoskeleton and its regulation by calcium and protons // Plant Physiol. - 2016. - V. 170. - P. 3-22.

4. Kovaleva L., Zakharova E. Hormonal status of the pollen-pistil system at the progamic phase of fertilization after compatible and incompatible pollination in Petunia hybrida L. // Sex Plant Reprod. - 2003. - V. 16. - P. 191-196.

5. Kovaleva L.V., Zakharova E.V., Minkina Y.V., Voronkov A.S. Effects of flavonols and phytohormones on germination and growth of petunia male gametophyte // Allelopathy J. - 2009. - V. 23. - P. 51-62.

6. Kovaleva L.V., Voronkov A.S., Zakharova E.V. Role of auxin and cytokinin in the regulation of the actin cytoskeleton in the vitro germinating male gametophyte of petunia // Russ J. Plant Physiol. - 2015. - V. 62. - P. 179-186.

7. Lang V., Pertl-Obermeyer H., Safiarian M.J., Obermeyer G. Pump up the volume - a central role for the plasma membrane $\mathrm{H}(+)$ pump in pollen germination and tube growth // Protoplasma. - 2014. - V. 251. - P. 477-488.

8. Certal A.C., Almeida R.B., Carvalho L.M., Wong E., Moreno N., Michard E., Carmeiro J., Rodriguezleon J., Wu H.M., Cheung A.Y., Feijo J.A. Exclusion of a proton ATPase from the apical membrane is associated with cell polarity and tip growth in Nicotiana tabacum pollen tubes // Plant Cell. - 2008. - V. 20. - P. 614-634.

9. Grabski S., Schindler M. Auxins and cytokinins as antipodal modulators of elasticity within the actin network of plant cells // Plant Physiol. - 1996. - V. 110. - P. 965-970.

KOVALEVA L.V. ${ }^{1}$, VORONKOV A.S. ${ }^{1}$, ZAKHAROVA E.V. ${ }^{2}$, TIMOFEEVA G.V. ${ }^{1}$

${ }^{1}$ Timiryazev Institute of Plant Physiology RAS,

Russia, Moscow, Botanicheskaya, 35

${ }^{2}$ Dep. of Genetics, Biotechnology, Plant Breeding \& Seed Science, Timiryazev Agricultural Academy,

Russia, Moscow

\section{PM $\mathrm{H}^{+}$-ATPase IS A POTENTIAL TARGET OF HORMONE SIGNALING DURING POLLEN TUBE GROWTH IN PETUNIA}

Aim. Pollen tube growth is the best known example of highly polarized plant cell expansion. This process is known to require intensive exocytosis at the tip which is supported by dynamic cytoskeleton, vesicle trafficking, and functioning of ion transporters in pollen tube plasma membrane (PM). We have attempted to test the possibility of plant hormones functioning as signal molecules in progamic phase of fertilization. Methods. The effects of exogenous plant hormones, indole-3-acetic acid (IAA), abscisic acid (ABA), gibberellin $\mathrm{A}_{3}\left(\mathrm{GA}_{3}\right)$, and cytokinin (kinetin) on the growth, PM polarization of in vitro germinating petunia (Petunia hybrida L.) pollen tubes were investigated. Results. Growthstimulated effects of IAA, $\mathrm{ABA}$ and $\mathrm{GA}_{3}$ were accompanied by orthovanadate-sensitive hyperpolarization of PM suggesting possible involvement of $\mathrm{PM} \mathrm{H}^{+}$-ATPase activation in this process. Fluorescent labeling the enzyme with $\mathrm{H}^{+}$ATPase antibodies exhibited IAA- and ABA-induced lateral membrane redistribution of it into the subapical zone of pollen tube PM. Conclusions. All these results, taken together, indicate the potential target of hormone signaling in pollen tubes, $\mathrm{PM} \mathrm{H}^{+}$-ATPase, related to the hormone-induced modulation of the above mentioned characteristics of this proton pump.

Keywords: Petunia hybrida, Pollen tube, $\mathrm{PM} \mathrm{H}^{+}$-ATPase, phytohormones. 